\title{
THE SUMMER MEETING IN TORONTO
}

The American Mathematical Society held its seventy-second summer meeting at Toronto, Ontario, from Tuesday through Friday, August 29-September 1, 1967. All sessions were held on the campus of the University of Toronto. About 2,184 persons attended, including about 1000 members of the Society.

Professor Samuel Eilenberg of Columbia University delivered the Colloquium Lectures on Universal algebra and the theory of automata. He was introduced by Professors C. B. Morrey, Jr., S. MacLane, N. Steenrod and F. W. Lawvere.

By invitation of the Committee to Select Hour Speakers for Summer and Annual Meetings, Professor David Gale of the University of California spoke on $A$ mathematical theory of optimal economic development at a joint session with the Econometric Society, chaired by Professor L. McKenzie, and Professor Michael Artin of the Massachusetts Institute of Technology spoke on The topology of algebraic varieties, with Professor R. M. Thrall presiding.

There were 26 sessions for contributed papers, at which 196 papers were presented. The chairmen of these sessions were Professors J. Aczel, M. G. Arsove, R. Blum, D. Borwein, W. C. Brown, R. H. Cole, H. S. M. Coxeter, C. Davis, G. F. D. Duff, G. A. Grätzer, R. K. Guy, P. R. Halmos, P. C. Hammer, R. Harrop, H. A. Heilbronn, E. Hemmingsen, T. Husain, L. Janos, S. A. Jennings, W. M. Kahan, P. Lancaster, L. Lorch, G. G. Lorentz, D. R. McMillan, P. R. Meyer, E. A. Michael, T. Norvig, V. V. Rao, D. C. Russell, D. Sarafyan, P. P. Saworotnow, D. A. Sprott, R. G. Stanton, B. M. Stewart, R. M. Thrall, W. T. Tutte, L. C. Young.

This meeting was held in conjunction with meetings of the Mathematical Association of America, the Society for Industrial and Applied Mathematics, the Canadian Mathematical Congress, Pi Mu Epsilon and Mu Alpha Theta. Professor G. C. Rota of Rockefeller University delivered the Hedrick Lectures of the Association on Combinatorial analysis as a theory. Professor J. Lehner of the University of Maryland spoke on The Picard Theorem, and Professor P. Samuel of the University of Paris spoke on Unique factorization. Professor C. C. Lin of the Massachusetts Institute of Technology presented the S.I.A.M. von Neumann Lecture on "Some aspects of stellar dynamics and galactic structure." Professor H. S. M. Coxeter of the University of Toronto delivered the Presidential Address of the Canadian Mathematical Congress on The problem of Apollonius. 
The Council met on August 29 at 5:00 P.M.

The Secretary announced the election of the following two hundred forty-six persons to ordinary membership in the Society:

Abshalom, Mizrahi, Indiana University;

Adamski, Ramona M., Purdue University;

Adler, David N., Adelphi University;

Alin, John S., University of Nebraska;

Allen, Daniel L., General Motors Corporation, Warren, Michigan;

Alter, Nicholas, Robert College, Istanbul, Turkey;

Arriaga, Rodrick M., University of California, Davis;

Asadulla, Syed, Miami University;

Austin, John A., Jr., Virginia Military Institute;

Ax, George B., Virginia Military Institute;

Badhwar, Lalit K., Computing Devices of Canada, Ottawa, Ontario, Canada;

Baird, Robert J., Wayne State University;

Batt, Jurgen O., Kent State University;

Berger, Emil J., St. Paul Public Schools, St. Paul, Minnesota;

Bichteler, Klaus R., Southwest Center for Advanced Studies, Dallas, Texas;

Birman, Joan L., New York University-Courant;

Bond, William T., Bendix Radio Division, Baltimore, Maryland;

Boorman, Scott A., Harvard University;

Brown, Alfred J., Wayne State University;

Bradford, Giles Edward III, University of Texas;

Breuer, Manfred, University of Kansas;

Bright, George W., Rice University;

Bubeck, John D. M., Cathedral Science Associates, Schuylkill Haven, Pennsylvania;

Bunce, John W., Tulane University;

Burdoin, Allen D., Milton Academy;

Burenkov, Viktor I., Steklov Institute of Mathematics, Moscow V-333, U.S.S.R.;

Busenberg, Stavros N., Loyola University;

Buser, Sister Mary Paul, Marymount College;

Cable, Charles A., Juniata College;

Cameron, Theodore G., Radio Corporation of American, Camden, New Jersey;

Carlson, David H., Case Western Reserve University;

Carlson, Carl G. R., Worcester Polytechnic Institute;

Chan, Sui Wah, University of Zurich, Zurich, Switzerland;

Chang, Ping-Tung, Mt. St. Paul College;

Chowla, Paromita, Pennsylvania State University;

Coats, Gary L., University of New Mexico;

Coleman, William P., Auburn University;

Crosby, Jack W., University of Houston;

Crown, Gary D., Western Michigan University;

Deely, John J., Sandia Corporation, Albuquerque, New Mexico;

De La Penha, Guilherme M., University of Catolica, Rio de Janeiro, Brazil;

Denes, Edward J. Jr., Kathleen Laycock Country Day School, Green Farms, Connecticut;

Dodds, Reginald M., University of Stellenbosch, Stellenbosch, South Africa;

Donaghy, Charles M., Pride Transportation Incorporated, Brooklyn, New York;

Driscoll, Sister Brigid, Marymount College; 
Dunn, Judith Kay, University of North Carolina at Chapel Hill;

Eisenhart, Laurance D., Westinghouse Electric Company, Pittsburgh, Pennsylvania;

Elwin, John D., Oregon State University;

Federbush, Paul G., University of Michigan;

Feigelstock, Steven G., Brooklyn Polytechnic Institute;

Fennell, Robert E., University of Iowa;

Ferguson, Ronald D., Sam Houston High School, Arlington, Texas;

Finkelstein, Harold S., University of Colorado;

Fitzgerald, Carl H., Stanford University;

Flamboe, Ronald R., Brigham Young University;

Foote, Joe R., University of Missouri at Rolla;

Forest, Charles A., Los Alamos Scientific Laboratory, Los Alamos, New Mexico;

Forster, Gregory, International Business Machines Corporation, Louisville, Kentucky;

Foster, Howard J., Alabama A and M College;

Frady, Charles S., Georgia State College;

Friedlander, Richard J., University of California at Los Angeles;

Friedman, Harold L., Kaufmann and Friedman, Corona, New York;

Fulton, John D., Oak Ridge National Laboratories, Oak Ridge, Tennessee;

Gabriel, Leonard F., Corn Products Corporation, Argo, Illinois;

Gaskill, Jerry W., George Washington University;

Gaussens, Pierre, Electricite de France, 92 Clamart, France;

Geiger, William R., University of Maine;

Gendler, Joyce K., Pennsylvania State University;

Gerber, Harvey, Simon Fraser University;

Gerhard, J. Calvin, Western Michigan University;

Gibbs, Richard M., Hayes International Corporation, Birmingham, Alabama;

Gillespie, Thomas J., Chicago City College;

Goldberg, Sally Eileen, Investors Management, Elizabeth, New Jersey;

Golland, Ronald W., Illinois Institute of Technology, Research Institute;

Gordon, Milton A., Loyola University;

Gordon, Zulette L., University of Victoria;

Gormley, Robert E., Prudential Insurance Company, Newark, New Jersey;

Green, Robert L., Unitrode, Watertown, Massachusetts;

Greendlinger, Elena K., Chemical Engineering College, Ivanovo, U.S.S.R.;

Grimble, Helen B., Point Park College;

Guber, Walter, Mathematics Applied Group, White Plains, New York;

Guseman, Lawrence F., Jr., National Aeronautic and Space Admin., Houston, Texas;

Haborak, George E., U. S. Naval Academy;

Hajek, Otomar, Caroline University, Praha-Karlin, Czechoslovakia;

Hakala, Reino W., Michigan Technological University;

Hall, Veronica A., 142 East 33rd Street, New York, New York;

Handelsman, Richard A., Brown University;

Hanlon, William L., Ladycliff College;

Harris, Lawrence, New York University;

Hartman, Elliott, Grumman Aircraft Corporation, Bethpage, New York;

Harshbarger, Ronald J., Pennsylvania State University;

Harvey, William J., Columbia University;

Hayuki, Hiroshi, University of Waterloo; 
Herod, James V., Georgia Institute of Technology;

Herrmann, Robert A., American University;

Higgins, G. Albert, Jr., Mt. Hermon School, Mt. Hermon, Massachusetts;

Hilding, Stephen R., Gustavus Adolphus College;

Hingert, Herbert B., University of Toronto

Hodgson, Jonathan P. E., University of Pennyslvania;

Hoff man, Martin S., Arizona State University;

Huggins, Marietta S., Univac, St. Paul, Minnesota;

Hughes, Carl C., Kentucky Southern College;

Hull, Richard G., SUNY at Buffalo;

Jandy, Geza, University of Economic Science, Budapest, Hungary;

Jensen, Jens A., University of Wyoming;

Jones, Constance J., Trinity University;

Kagiwada, Harriet N., Rand Corporation, Santa Monica, California;

Kanchanavasita, Prasert, Royal Military Academy, Bangkok, Thailand;

Keiser, Victor H., Whitman College;

Keun, William J., Lambton College;

King, Joseph F., U. S. Army, Ent Air Force Base, Colorado;

Kriegsman, Helen F., Kansas State College;

Krier, Nicholas K., Ohio State University;

Kshirsagar, Shrirang M., 1952 East Allegheny Avenue, Philadelphia, Pennsylvania;

Kuczura, Anatol, Bell Telephone Company, Holmdel, New Jersey;

Kyriazopoulos, Dimitrios G., DeVry Technical Institute;

Lakein, Richard B., University of Maryland;

Lambarth, David A., U. S. Air Force, Plattsburgh Air Force Base, New York;

Lamphere, Robert L., Triton College;

Larson, Larrie J., Dugway Proving Ground, Meteorology Building, Dugway Proving

Ground, Utah;

Lawruk, Bohdan, McGill University;

Lazer, Alan C., Case-Western Reserve University;

Lieberman, Alfred G., Johns Hopkins University;

Lieberman, Burton B., Brooklyn Polytechnic Institute;

Liebert, Wolfgang J., New Mexico State University;

Lindahl, Robert J., Pennsylvania State University;

Lindstrum, Andrew O., Jr., Southern Illinois University;

Lodhi, A. Sattar Khan, Paul Quinn College;

Loring, Ronald J., State College at Boston;

Lovelock, David, University of South Africa, Pretoria, South Africa;

Lucas, Harry, Jr., 850 Houston Club Building, Houston, Texas;

Ludwig, Garry, University of Alberta;

Malanga, George P., Rutgers, The State University;

Manez, Elisa L., St. Xavier College;

Mann, Avino'Am, University of Illinois;

Mann, James T., Ohio State University;

Marcus, Robert I., New York University;

Marley, Gerald C., University of Arizona;

Marshall, Robert R., University of Utah;

Martin, Clyde F., University of Wyoming;

Matucha, John L., University of California at Davis;

Matula, David W., Washington University; 
Mayes, Vivienne M., Baylor University;

McBride, Elna B., Memphis State University;

McElroy, Melvin L., International Business Machines Corporation, Seattle, Washington;

McJunkin, Martha M., Warner Towers, Alexandria, Virginia;

McMillan, John E., University of Wisconsin-Milwaukee;

Mischel, Cynthia R., University of California at Davis;

Montgomery, Emily D., Lee College;

Mooney, Michael C., University of Georgia;

Moore, Charles E., U. S. Naval Academy;

Moore, Ronald L., Roanoke College;

Moran, Bryan P., University of Notre Dame;

Morand, Guy D., Transit Authority, 370 Jay Street, New York, New York;

Mularkey, Sister Mary Kenneth, St. Xavier College;

Mulryan, Michael J., Adelphi University;

Murphy, Thomas N., Trent University;

Myers, Sister Mary Felicitas, Trocaire College;

Newstead, Peter E., Harvard University;

Nigam, Ishwar S., University of Georgia;

Nowlan, Robert A., St. Mary's College;

Oberhoff, Kenneth Earl, University of Houston;

Ortner, Gene M., Michigan Technological University;

Osher, Stanley J., Brookhaven National Laboratory;

Ossessia, Michel G., Clarion State College;

Ozsvath, Istvan, Southwest Center for Advance Studies;

Palmer, L. Bruce, U. S. Naval Academy;

Peters, Franz, St. John's University;

Petersson, Holger, P., University of Iowa;

Pincus, Martin M., Brooklyn Polytechnic Institute;

Piron, Georges, St. Louis University, Baguio City, Philippines;

Platt, Craig R., Pennsylvania State University;

Pokropp, Fritz, University of Cincinnati;

Poland, John C., Carleton University;

Pomfret, James C., State University of New York, College at Oswego;

Porter, Than R., New York University-Courant;

Powell, Steven F., Lenoir County Community College;

Queen, William C., SUNY at Stony Brook;

Quirk, Paul B., University of Georgia;

Radu, Rosca, Academy R.S.R.-Institute of Mathematics, Bucharest, Rumania;

Randack, Jerry F., III, University of Albuquerque;

Reed, Kennard W., Jr., Georgetown University;

Reid, Lois J., Duke University;

Reudink, Douglas O., Bell Telephone Company, Crawford Hill, New Jersey;

Reves, George E., The Citadel;

Rhodes, Joe B., University of Texas;

Rhodes, John L., University of California at Berkeley;

Riley, David R., University of California at Santa Barbara;

Roberts, Maria D., University of California at Berkeley;

Rodemich, Eugene R., California Institute of Technology;

Romero, Lazaro M., U. S. Bureau of Reclamation, Montrose, Colorado; 
Rosenberg, David, University of Tennessee;

Rosenberg, Gabriel, Queens College (CUNY);

Ross, Palma L., University of Texas at El Paso;

Ross, Roderick A., University of Toronto;

Rousseau, George H., University of Leicester, Leicester, England;

Sager, David G., Johns Hopkins University;

Sarkany, Endre F., University of Missouri at Rolla;

Sanders, Oliver Luckett, Box 148, Gila Bend, Arizona;

Sasso, Leonard P., Jr., University of California at Berkeley;

Sawtelle, Peter G., University of Maine;

Scheitlin, Alexander Guy, New York University-Courant;

Schillinger, Sister M. Estelle, Good Counsel College;

Schirmer, Helga H., Carleton University;

Schneider, Edgar R., International Business Machines Corporation, Ft. Walton Beach, Florida;

Schoenfeld, Peter S., Howard University;

Schuur, Susan E., Michigan State University;

Schweber, Ellena K., City College (CUNY);

Sedlock, James T., University of Connecticut;

Sharp, Edward A., Creighton University;

Shin, Yong-Tae, Queen's University;

Shumate, Ervin H., Jr., U. S. Army, Fort George G. Meade, Maryland;

Sibilia, Sister Anita, Villa Walsh College;

Skala, Helen, Illinois Institute of Technology;

Small, Donald B., University of Connecticut;

Smith, Joseph A., Chicago City Junior College;

Snygg, Charles E., University of Michigan;

Soofi, Abdul R., Ottawa University;

Sorrells, Roger, San Antonio College;

Sotomayor, Jorge, University of California at Berkeley;

Spain, Harry E., University of Michigan;

Stokes, H. Christine, University of Mississippi;

Summers, William H., Louisiana State University;

Tam, Kwok-Wai, Portland State College;

Taylor, Laird E., Stanford University;

Taylor, Theodore B., Harvard University, Computing Center;

Terry, Betty C., University of California at Davis;

Thomas, James W., Southern Illinois University;

Tjurin, Andrein, Steklov Institute of Mathematics, Moscow V-333, U.S.S.R.;

Todorov, Pavel G., University of Plovdiv, Plovdiv, Bulgaria;

Travis, Raymond D., University of Detroit;

Tripp, Leonard L., Brigham Young University;

Vandenburch, William G., California State College at Hayward;

Vitale, Vincent G., New York University;

Vagts, Arnold E., Jr., Systems Development Corporation, Dover-Foxcroft, Maine;

Veal, Frances, Douglas Aircraft Corporation, Santa Monica, California;

Walczak, Hubert R., College of St. Thomas;

Walling, Derald D., Texas Technological College;

White, Anthony G., University of Delaware;

Whitlock, Prentice E., Jersey City State College; 
Whitney, James N., Brandeis University;

Wilbur, W. John, University of California at Davis;

Williams, Bennie B., University of Texas at Arlington;

Williams, Velma M., Texas Southern University;

Witten, Louis, RIAS, Baltimore, Maryland;

Yates, Wilma L., Stetson University;

The following eight hundred and eight persons have been elected to membership in the Society on nomination of institutional members as indicated:

Adelphi University: Neoptolemos Cleopa, Alan W. Hulsaver, Charles R. Katholi.

University of Alberta: Ivan Baggs, Edward R. Graff, Denis Hanson, Rudolph Hrycay, Yong-Woon Kim.

Andrews University: Donald J. Albers, Kenneth E. Thomas.

Antioch College: Richard Bryan Amundson, Bruce A. Wolk.

Arizona State University: Gordon O. Berg, Henrik Bresinski, Charles L. Hagopian. University of Arizona: Ronald R. Miller, Jimmie Baker Smith, James W. Thomas.

University of Arkansas: James B. Barksdale, Jr., Russell G. Brasher, Albert G. Starling.

Brandeis University: Mark Bridger, Daniel Comenetz, Karel Drbohlav, Robert

Goren, Martha A. Jaffe, Larry E. Johnson, David B. Merdith, Mark B. Ramras,

Sevin P. Recillas, Mark J. Schmidt, Suzanne Treves, Karen Uhlenbeck, Reginald $M$. Wood.

University of British Columbia: David M. Foster, Joel Hillel, Hwei-Mei Ko, Edward R. Powell, Kenneth W. Welsh.

Brown University: Howard M. Cohen, Edward A. Grove, Alfred P. Hallstrom, David H. Issac, Alan E. Rosenberg, Steven L. Shrier, Stephen J. Tillman, Michael D. Weiss.

University of California, Berkeley: Dennis P. Allen, Jr., Mohamed A. Amer, Robert Arnold, Ruben G. Babayan, Raul Bravo, William T. Bryan, James R. Byers, Shih-Hung Chang, Elon Ryder Close, Stephen A. Coffin, Thomas J. Cook, Peter W. Cressman, Andre KeKorvin, H. Robert Downs, Charles R. Eckel, Donald A. Eisenman, Kenneth L. Fields, John M. Frands, Julia Frandsen, Gary L. Ganske, Jonathan Goldstine, Thomas C. Green, John M. Grover, Stephen H. Hechler, John M. Hosack, Brent L. Jackson, Thomas F. Jambois, Gary R. Jensen, John N. Johnson, Olin G. Johnson, Mohammad A. Kahn, Ludwig A. Khatchatooriantz, James R. King, Arthur J. Krener, Myung He. S. Kwack, Michael K. Kwatinetz, John O. Lacy, Richard N. Lane, James B. Leatherman, Warren K. Leffler, Yung-Chen Lu, Puey-Chong Lum, William J. Mitchell, Hiroko Miyama, John D. Moore, Amy C. Murray, David H. G. Nash, Hussain S. Nur, Don L. Pigozzi, William G. Poole, Jr., Russell E. Powell, Alwyn D. Randall, Diane Resek, Donald B. Richert, Peter Ross, Sherwood Samn, Walter J. Savitch, James H. Schmerl, Wilfried Schmid, Sidney C. Scull, Ralph L. Seifert, Jr., Siavash M. Shahshahani, James R. Shilleto, Norman L. Siever, Vashishtha N. Singh, David Skurnick, Stephen A. Sokolow, Richard P. Stauduhar, Howard B. Stauffer, Price E. Stiffler, Jr., Howard S. G. Swann, David S. Tartakoff, Francisco J. Thayer, Per Tomter, John Van Eps, Diane M. Wagner, Ralph E. Walde, Cecilia W. Wang, Norris F. Weaver, Patrick P. Weidhaas, Peter J. Weinberger, Gerald C. Wolpin, Yechezkel Zalcstein, John R. Zumbrunn.

University of California, Davis: Alfred L. Levinson. 
University of California, Los Angeles: Ira B. Russak.

University of California, Riverside: John M. Bownds, Johnny A. Johnson, Tjoioe H. Kwan, Edward D. Shirley, William R. Wade, II.

University of California, Santa Barbara: Willett Ware, III.

California Institute of Technology: Daniel L. Davis.

California State College at Fullerton: Robert J. Juergens.

California State College at Hayward: John M. Jefferson, James S. Mcintyre, Hal Sudborough.

Carnegie-Mellon University: Theodore J. Gifford, William T. Kraynek, David K. McLain, Harry T. Sedinger.

Case Western Reserve University: Shair Ahmad, Yash P. Singh.

Central Michigan University: James K. Bidwell, Dean O. Hinshaw.

University of Chicago: William C. Connett, Albert F. Lawrence, Marie-Louise Welland.

Clarkson College of Technology: Dale W. Kreisler, Matthew W. Swinsick, Michael A. Grajek.

Clemson University: Donald M. Bardwell, Patrick C. Bowie, Harold B. Reiter.

University of Colorado: Mangho Ahuga, Peter Bancroft, Thomas H. Jefferson, Jr., Sudershan Kapoor, Leslie E. Shader, Robert I. Snell.

Columbia University: Alvin Z. Hornstein, John E. Jayne, Arnold D. Kleinstein, John R. Liukkonen, Donald H. Singley, Michael R. Stein, Miyata Takehiko, ChakKuen Wong.

Dalhousie University: Edward J. Lewis, Arthur G. Thomas, John A. Wright.

University of Dayton: Donald J. Marxen, John J. Sikora, Gerard O. Wunderly.

Drexel Institute of Technology: Michael S. Borowsky, Robert C. Busby, Sylvan S. Pinsky.

Emory and Henry College: Walter T. Graybeal.

F. T. Nicholls State College: Larry S. Haw, Merlin M. Ohmer.

Fairleigh Dickinson University: Hussein A. Elkholy.

Fordham University: Kenneth F. Bailie, James J. Hamilton, Terence J. O'Hara.

Frederick College: John Dauchtry, John Fogelgren, Jeryl Seaman.

Georgia Institute of Technology: Stanely J. Wertheimer.

Georgetown University: Thomas P. Haggerty, John B. Manning, Ann M. Meyers.

Harvey Mudd College: Roger C. Ray.

Hope College: Jay E. Folkert.

University of Houston: William C. Haase, C. Wayne Proctor.

Idaho State University: Keith R. Brown, Michael A. Lintner, Henry L. Thaggert.

Illinois State University: Jackson N. Henry, James J. Higgins, Thomas E. Yerkey. University of Illinois: Joseph A. Applebaum, Charles W. Conatser, Kenneth A. Bowen, Jean H. Cook, Barbara K. Falkner, Patrica A. Fordell, Marshall M. Fraser, Brayton I. Gray, Peter R. Hall, Martin R. Hoffman, Claire E. Krukenberg, Keiko Kudo, Robert E. Lewis, Marjorie A. Moretz, Charles W. Neville, Theodore T. Papas, Frederick C. Piper, Daniel Richardson, Surjit Singh, Ellen M. Torrance, Robert L. Ward, Waldemar C. Weber, Muhammad S. Abu-Salih.

Iowa State University: John M. Clark, Douglas W. Curtis, Michael L. Engquist, Robert A. McCoy.

University of Iowa: William D. Royalty.

John Hopkins University: Michael R. Ackerman, John L. Greenberg, Liang-Shin Hahn, Chin-Chin Lan, R. Gwillim Law, Jr., Peter Wallner. 
University of Kentucky: Nancy M. Dykes, Richard A. Mukkikin, James D. Powell, Selden Y. Trimble, James E. Weatherbee, Donald J. Wright.

Knox College: Joseph L. Cecchi, David M. Slocum, Ronald J. Stern.

Lehigh University: Daniel J. Fleming.

Macalester College: Richard D. Hurwitz, Gerald R. Martin.

University of Maine: James F. Calarco, Wayne E. Carter, Jan Erik H. Johansson. Marquette University: George L. Edenharder, Mary L. Funke, Thomas Neavins.

University of Maryland: Earl R. Barnes, Stephen R. Bernfeld, Joseph R. Caspar, Jim M. Cushing, Richard M. Elkin, Lawrence E. Levine, Ewing L. Lusk, David L. McNeill, Stanley J. Poreda, Edward B. Saff, Robert J. Seid, George D. Shank, Arthur B. Stephens, Robert S. Stepleman, Hugo S. H. Sun, Elliot Winston.

Massachusetts Institute of Technology: Melvin Band, David A. Beer, Earl D. Brown, Bennett Eisenberg, Stanley A. Erickson, Jr., Lawrence Feiner, Michael P. Froman, Jay E. Israel, Hershy H. Kisilvesky, Larry L. Langdon, Michael J. J. Lennon, Ronald L. Lipsman, John M. MacIntyre, Carey A. Mann, Jr., Joel Moses, Stephen J. Rallis, David P. Robbins, Sandow S. Ruby, Stephen L. Williams, Lawrence A. Zalcman.

University of Massachusetts: Lawrence E. McKnight.

McGill University: Gordon R. Mason.

McMaster University: Donald E. Eastman, Barry W. Fawcett, James A. Gerhard, Evelyn R. Nelson, Claire W. Zeller.

University of Missouri: Maurice J. Dupre, Alan L. Lambert, John J. Leeson.

University of Michigan: Edward M. Bloombert, Robert Connelly, Jr., William A. Groening, Charles M. Schneeberger, Stephen B. Seidman, Johanna Stenzel, Martin C. Weinrich.

Michigan State University: Harold A. Allen, Gail H. Atneosen, David J. Bernstein, Chang Choi, Arthur G. Conn, William M. Conner, John K. Cooper, William F. Cutlip, Wayne C. Ellis, Norman J. Frisch, David E. Galewski, Leonard J. Harding, Jr., William L. Hightower, Jeffrey L. Johnson, Ronnie Lee, Richard T. Miller, Paul S. Muhly, Clive M. Reis, James W. Richards, Dennis M. Roseman, Richard J. Tondra, James E. Van Deventer, Paul B. Venzke, James R. Weaver.

University of Minnesota: George H. Bridgman, Halldor T. Gudjonsson, Bernard N. Harvey, Alexia A. Henderson, John R. Higgins, Dewayne C. Hillman, Samiha S. Ibrahim, Samuel John Klippenstein, John S. Kronholm, John A. Little, Dahlard L. Lukes, James H. Olsen, Willis L. Owen, Jr., J. Eli Rosenfield, Mahmoud Sayrafiezadeh, Laurence Weinberg.

University of Mississippi: Thomas M. Phillips, Dean B. Priest, Harry A. Watson, Jr. University of Missouri: Chi Cheng Chen, Larry W. Davis, Feng Yan Kwang, Raymond Morgan, Jr.

Montana State University: Kent F. Carlson.

University of Montana: John T. Clinker, Elmer W. Gall, Charles M. Greene, Bernard W. Irlbeck, James M. Lucke, Thomas L. Munkres, Denny C. Pilling, Ronald K. Wade.

University of Nebraska: Stanley B. Eliason, Glenn L. Pfeifer, Warren E. Shreve.

University of Nevada: Kathleen H. Galloway, David A. Seagraves.

University of New Hampshire: Raymond A. Beauregard, Charles M. Biles, Halsey Stickel, Jr.

Eastern New Mexico University: Constantine G. Cassapakis, Dennis M. Kulvicki, Jerry F. Rigdon. 
Nerw Mexico State University: James A. Davis, Frieda A. Koster, Larry D. Parker, Billy J. Thorne, Michael J. Wetzel.

New York University-Courant Institute of Mathematical Sciences: Boruch Baumslag, Richard N. Cain, Milos A. Dostal, Alan T. Gray, Mohammed A. Gwaiz, Edward P. Helpert, Howard J. Jacobwitz, Stephen I. Jasik, Hermann R. Karcher, Barbara L. Keyfitz, David G. Korn, James M. McHugh, Thomas Y. MottSmith, Lon Rosen, John G. Stevens, Jay Turim, Eli L. Turkel, Oscar Urreiztieta, Remi Vaillancourt, John W. Voegel.

University of North Carolina: Stanley R. Clemens, Charles D. Cunningham, Kirk A. Griffen, David E. Radford, Barbara S. Shively, Robert C. Shock, Anita H. Somers, Samuel A. Truitt, Jr., William T. Whitley, Thomas B. Williams.

University of North Carolina, Greensboro: Elizabeth A. Beamer, Evelyn F. Johnson, Linda L. Smith.

North Carolina State University at Raleigh: George M. Eargle, Billy J. Gilpin, Lynn M. Perry, Jr.

University of North Dakota: David S. Lenaburg, Walter E. S. Will, Peter A. Willyard. North Texas State University: Howard L. Penn, Samuel A. Rodgers, Charles I. Votaw.

Northern Michigan University: John C. Comfort.

Northwestern University: William C. Brown, Jr., Sou-Yung Chiu, Amassa C. Fauntleroy, Robert B. Feinberg, Christopher A. Field, Joseph W. Paciorek, Min Ming Tang.

University of Notre Dame: Martin H. Dull, Rene R. Gaudreau, Charles H. Jepsen, Thomas E. McCormick, III, Michael R. Meck.

Ohio State University: Patrick J. Caulfield, Ronald A. DeVore, Guy T. Hogan, Marc W. Konvisser.

Ohio University: Stuart R. Black, Edward L. Davis, Ronald E. Hall, Lewis T. Redeker.

Ohio Wesleyan University: Marjorie E. Ferster, Arthur K. Peacock, Deborah J. Wagner.

University of Oklahoma: Stanley N. Burris, William J. Frawley, David K. Hughes.

Oklahoma State University: Freddie E. Tidmore, James H. Yates.

University of Oregon: William J. Carsrud, Arthur J. Clemons, Joel L. Cunningham, Robert E. Dressler, Jerry B. Eagle, Casey C. Fast, Howard R. Gage, Richard R. Hallett, Wayland M. Hubbart, Douglas H. Kelker, Jeanne LaDuke, Daniel G. Lamet, Martin L. Osborne, Julius A. Minsky, Walter A. Petersen, William E. Pfaffenberger, Ralph G. Pond, Forrest A. Richen, Roy W. Ryden, III, John H. Schultz, Jr., Steven M. Simmons, Thomas A. Swanson, Ronald L. Wagoner.

Oregon State University: William Margolis.

University of Ottawa: Koo Guan Choo, Pak Ken Wong.

Pacific Lutheran University: Linda Connolly, Paul J. Olsen.

Pennsylvania State University: Anthony J. D'Aristotle, Morris J. Deleon, Douglas H. Frank, Sharadchandra W. Joshi, Gerard J. Lallement, Francisco L. Marin, Phillys E. McNeil, Robert B. McNeill, Martha H. Porter, Sahib Singh, Robert S. Smith, Robert M. Vancko.

Portland State College: John D. Daily, Barbara R. Jeffcott.

Princeton University: Reynir Axelsson, Sylvain E. Cappell, Richard A. Graff, Raphp Greenberg, Louis H. Kauffman, Masao Kishore, Paul G. Machuca, Jeffrey L. Needleman, Joseph A. Neisendorfer, Stanley Ocken, Harsh V. Pittie, Brian T. Smith, Albert L. Vitter, III, Robert L. Walton, Thomas R. Wolfe. 
Purdue University: Harry J. Carr, Kenneth J. Danhof, Michael J. Dixon, Larry $Q$. Eifler, David C. Haddad, Gary M. Heggard, Men-Fon Huang, Hans F. Langmaack, Fon-Che Liu, Calvin J. Mannes, Umesh P. Nagarkatte, Herbert Popp, Lawrence A. Shulman, Shih-Liang Wen, Robert D. Williams.

Queen's College CUNY: Diane J. Dupont, William F. Dwyer, Mona Fabricant.

Queen's University: Sydney D. Bulman-Fleming, Robin Giles, Sribatsa Nanda, T. Rolf Turner, Michael J. Wilmut.

Rensselaer Polytechnic Institute: Paul Levy, Arthur L. Schoenstadt, Lee J. Supowit. University of Rhode Island: Robert A. Barron, Norman J. Finizo, Chris P. Tsokos. Rice University: Edward W. Formanek, Ushi I. Huang, David C. Shreve, David J. Vanderschel, Clarence W. Wilkerson, Jr.

University of Rochester: Mario C. DeMatos, John G. Michaels, Balmohan V. Limaye. Rockefeller University: Richard M. Caron, Robert J. Donoghey, Franciser A. Grunbaum, Patrick E. O'Neil.

Rockford College: Thomas A. Chapman, Carolyn J. Mattila.

Rutgers, The State University: John D. Baildon, Victor P. Camillo, Harry S. Cash, Floyd B. Cole, III, Peter J. Evanovich, Charles B. Hallahan, William E. Haver, Carl P. Pixley, Thomas E. Williamson, Jr.

Sacramento State College: Richard E. Breverly, William K. Schwarze.

San Diego State College: Gordon Whitnall.

San Francisco State College: Beverly N. Kraai, David J. Fontana, Wesley W. Tom. University of Santa Clara: Gary G. Ford, Bruce D. McLemore, Russell D. Mereclith. University of Saskatchewan: Andrew Fedorus, Dallas J. Howe, Robb E. Smith.

Simon Fraser University: Robert McKeever.

South Dakota School of Mines and Technology: Gary L. Britton, Garth A. P. Peterson, J. Suitt William.

South Illinois University, Carbondale: Aalt Dijksma, John V. Erhart, Lynn H. Ripper, Robert P. Sealy.

Southern Illinois University, Edwardsville,: Lyman S Holden.

Southern Methodist University: William D. Corder, Charles W. Taylor, Patricia E. Wood.

Southwest Texas State College: Gerald F. Busald, Raymond W. Czichos, Gerald R. Stewart.

Stanford University: Grant P. Brown, Stanley C. Bisenstat, Ronald E. Glaser, David M. Green, Allen E. Hatcher, Charles N. Johnson, Terry C. Lawson, Norman D. Neff, Esther G. Portnoy, Masakazu Takahashi, David W. Wigner, Robert S. Wolf, Jeffery I. Zucker.

State College at Salem: Robert V. Andersen.

Stephen F. Austin State College: John L. Gizelar, Ennis D. McCune.

Stevens Institute of Technology: Hugh M. Hilden, Joel D. Kagan, John J. Santa Pietro.

SUNY at Binghamton: Thomas W. Williams.

SUNY at Buffalo: Thomas L. Bartlow, Robert J. Knowles, Ro Neela, Akella L. Somayajulu.

St. Mary's College of Maryland: Miriam L. Long, Hassan Rabii-Nouri.

Saint Olaf College: James K. Ellickson, Peyton J. Huffman, Peggy L. Reinking. Suffolk University: Yonghyo H. Moon, Rosalind L. Nadeau.

Syracuse University: Daniel P. Doud, Philip B. Downum, John A. Kroulier, Gary M.

Sampson, Allen W. Weitsman.

Temple University: Herbert I. Brown. 
University of Tennessee: John L. Bailey, Judith K. Brinkley.

Texas Christian University: Paul J. Allen, Jr., George Berzsenye, James R. Boone, David L. Drennan, Michael E. Lord.

Texas Technical College: Roy D. Alston, Lyons H. Lockhart, Jr., Joe B. Thrash, Jr. University of Texas: Johnnie W. Baker, Peter A. Businger, Charles H..Farmer, Lee M. Johnson, Sister M. Assumption Molloy, David A. Senechalle, Boyce L. Turlington, Joseph F. Vance, Robert W. Vargas, Larry W. Wilson.

University of Toledo: Emman C. Obi, Rimantas A. Repsys.

University of Toronto: David F. Andrews, John C. Fisher, Bruce C. Gillian, David Jacobson, Andrew J. Kalotay, Hans Levenbach, Ved Perkash Madan, S. Manickam, Paul Milnes, Ralph A. Park, Leonard M. Steinberg, Frank W. Tucker.

Trinity College: Allen R. Elstein.

Tulane University: Frank H. Eckstein, Mary Catherine Flanders, Josephine S. Jolley, Alfred E. Tong.

University of Tulsa: Patrick L. Gore, Charles E. Lewis, Eldon N. Powers.

University of Utah: John R. Edwards, Jack W. Lamoreaux, Lois E. Mansfield, Richard R. Miller, Dix H. Pettey, Russell Wilhelmsen, James A. Wixom.

Vanderbilt University: Isaac S. Metts.

University of Virginia: Roger W. Allen, Jr., Berrien Moore, III, Frank S. Quinn, III, John W. Randolph, Raymond F. Snipes.

Washington University, St. Louis: Jonathan Cohen, Robert F. Emnett, Harvey C. Greenwald, Samuel B. Karp, Mu-Chou Liu, Dean G. Philps, Richard D. Weiner.

Washington and Lee University: Edward F. Turner.

University of Washington: David W. Barnette, Frank Castagna, Michael H. Clapp, Timothy E. Cramer, Peter Csontos, James P. Jones, Charles E. Murley, Frank H. Pollard, Cary B. Rader, Phillip Schultz, Charles I. Vinsonhaler, William J. Wichless, Jerry B. Williams.

Wayne State University: Harry Becker, Arvind B. Buche, Paul M. Gauthier, Heinrich Mantel, James J. Swanek, Samuel M. Zentman, William A. Vekovius.

Wellesley College: John A. Graham, Henry S. Lieberman.

Wesleyan University: William M. Baker, Clement L. Demayo, David B. Heisler, Ter-Jenq Huang, George D. Zahn.

West Virginia University: Joseph S. Cartisano, Paul Deskevich, David M. Moran.

Western Michigan University: Alan A. Bishop, Arlo D. Davis, Otis D. Shouse.

Western Washington State College: Gary L. Eerkes, Rodney D. Gentry, Peter W. Gray, James D. Harris.

Wheaton College: Pamela J. Taylor.

Wiley College: George A. Roberts, David W. Washington.

University of Wisconsin: Fredric D. Ancel, James E. Arnold, Jr., Michael G. Aschbacher, Martin W. Bartelt, Rochelle L. Boehning, Stephen S. Boyce, Robert L. Cannon, Jr., Frank A. Cezus, Richard C. Churchill, Robert L. Constable, Fred D. Crary, Joseph B. Dennin, Jr., David R. Ferguson, William G. Fortney, Solomon Garfunkel, J. Thomas Haigh, Henry W. Haslach, Jr., Larry D. Haugh, Laurence D. Hoffman, William H. Jaco, Stephen L. Jones, Robert G. Kribs, James J. Kuzmanovich, Donald A. Lawyer, Roy Y. Lee, John J. Libera, Jr., Shlomo Libeskind, Frank R. Loscalzo, Joseph Malkevitch, John H. Mason, William K. Mason, Stephen H. McCleary, Daniel L. McCord, Richard P. McGehee, David L. Morgan, Louise E. Moser, Harry C. Mullikin, Julius Nados, Edmund L. Ohorny, Michael Olinck, John E. Olson, Lynn M. Olson, Thomas J. Peneski, 
Keith R. Pierce, Gordon D. Prichett, John P. Quinn, Franklin B. Richards, Stanley J. Russell, William M. Self, Mark Sheingorn, Arnold C. Shilepsky, Kirby C. Smith, Robin N. Soloway, Phillip F. Stambaugh, Franklin D. Tall, David P. Vasholz, Wilbur J. Walkoe, Jr., Dallas E. Webster, David A. Weitzler, Rochelle L. Wilson, Jack Williamson, James M. Yohe.

Worcester Polytechnic Institute: Charles D. Konopka, Rodney W. Logan.

Yale University: Neal D. Glassman, Samuel R. Gordon, D. Randolph Johnson, Jr., Frank Kaplan, James M. Mann, Leonard L. Scott, Jr.

Yeshiva University: Joseph Bak, James S. Byranes, Anthony P. Ciszmazia, Robert P. Feinerman, Harold J. Gans, Noam Gordon, David N. Jacobson, H. Lee Michelson.

York University: J. Hardy Grant.

Xavier University: Kathleen S. Davidoff, John F. Niehaus.

It was announced that the following had been admitted to the Society in accordance with reciprocity agreements with various mathematical organizations:

Australian Mathematical Society: Roger B. Eggleton, Terence M. Gagen, Emmanuel Strzelecki.

Societe Mathematique de Belgium: Raoul F. Glodev, Marie Louise Vastersavendts. Calcutta Mathematical Society: Samir R. Majumdar.

Chinese Mathematical Society: Chih-Bing Ling.

Dansk Matematisk Forening: Anders J. Kock.

Deutsche Mathematiker Vereinigung: Bernhelm Booss, Munibur R. Chowdhury, Helmut Hasse, Herbert K. Meyer, Gert H. Huller, Hans-Joachim Nastold.

Societe Mathematique de France: Bui-Trong-Lieu, John A. Madore, Daniel Spohn.

Gesellschaft für Angewandte Mathematik und Mechanik: Hans F. Langmaack.

Indian Mathematical Society: Bhama R. Srinivasan, Ranjit S. Dhaliwal, Jeet M. Gandhi, Badrin Sahney, Darbari L. Sharma.

Israel Mathematical Union: Meira M. Lavie, Abraham Zaks.

Mathematical Society of Japan: Yoshikazu Karamatsu, Koji Katayama, Hideo Muto, Yasuo Nasu, Kiyoshi Nino, Hiroshi Noguchi, Kenjiro Okubo.

The London Mathematical Society: Arthur O. L. Atkin, Ian M. S. Dey, Henry R. Dowson, Howard Kleiman, David George Larman, Manojlo Maravic, Eric C. Milner, Frank Rhodes, Millu S. Rosanblatt-Roth, Bruce L. R. Shawyer, Dona P. Strauss, Peter Walters, Wlodzimierz Wrona.

Polskie Towarzystwo Matematyczne: Stanislau A. Golab.

Real Sociedad Mathematica Espanola: Gonzalo Sanchez-Vazquez, Juan S. San Roman. Suomen Matemaattinen Yhdistys: Veikko W. Nevanlinna.

Svenska Matematikersamfundet: Ake H. Samuelsson.

Unione Matematica Italina: Guiliano Sorani.

The following institutions have been elected to institutional membership:

Ithaca College, Ithaca, New York

York University, Toronto, Ontario, Canada

Suffolk University, Boston, Massachusetts

University of Arkansas, Fayetteville, Arkansas

University of North Dakota, Grand Forks, North Dakota 
North American Aviation, Inc., was elected to Corporate Membership.

The following Presidential appointments were announced:

Organizing Committee for the Summer Institute in Global Analysis (1968): F. Browder, S. S. Chern (co-chairman), Lars Hörmander, I. M. Singer, and Stephen Smale (co-chairman).

Added to the Committee to Monitor Problems in Communication: Jim Douglas, Jr., and Gordon L. Walker.

Organizing Committee for a Conference on Communication Problems in the Mathematical Sciences: H. A. Antosiewicz, F. Browder, Jim Douglas, Jr., H. Federer, W. J. LeVeque (chairman) C. B. Morrey, Jr., I. Niven, E. Pitcher, A. Rosenberg, G. L. Walker, F. J. Weyl.

Arrangements Committee for Conference on Communication Problems in the Mathematical Sciences (December 1967 in Providence): E. Pitcher, G. L. Walker (chairman).

Subcommittee on Lectures for the Committee on Mathematics in the Life Sciences: H. Bremermann, Murray Gerstenhaber (chairman), A. S. Householder, Paul Meier, R. M. Thrall.

Replacement on the Committee on Corporate Memberships: W. L. Duren, Jr. (occasioned by resignation of B. P. Gill).

Organizing Committee for a Symposium on Numerical Solution of Field Problems in Continuum Physics: Garrett Birkhoff (chairman).

The Secretary announced that the following persons represented the Society at various functions:

Joseph A. Sullivan at the inauguration of Burton Crosby Hallowell as President of Tufts University.

May R. Kinsolving at the inauguration of Robert W. Frederick, Jr. as President of Corning Community College.

Harold H. Hartzler at the inauguration of James F. Nickerson as President of Mankato State College.

Cecil B. Wright at the inauguration of Daniel Whitney Halliday as President of East Texas State University.

Flora Dinkines at the inauguration of John Alden Greenlee as President of the California State College at Los Angeles.

William M. Huebsch at the inauguration of Martin Meyerson as President of the State University of New York at Buffalo.

Frank L. Cleaver at the inauguration of John van Gaasbeek Elmendorf as President of New College.

E. B. Roessler at the presidential inauguration of Chico State College. 
Joel C. W. Rogers at the dedication of the Aerospace Laboratory of New York University.

The following persons have accepted invitations to address the Society at the times and places indicated:

$\begin{array}{lll}\text { Michael Artin } & \text { Toronto } & \text { August } 1967 \\ \text { Edgar H. Brown, Jr. } & \text { Cambridge } & \text { October } 1967 \\ \text { Arthur P. Mattuck } & \text { Cambridge } & \text { October 1967 } \\ \text { Alston S. Householder } & \text { Knoxville } & \text { November } 1967 \\ \text { Bjarni Jonsson } & \text { Knoxville } & \text { November } 1967 \\ \text { Pasquale Porcelli } & \text { Knoxville } & \text { November } 1967 \\ \text { Basil Gordon } & \text { Albuquerque } & \text { November } 1967 \\ \text { R. K. Getoor } & \text { Albuquerque } & \text { November } 1967 \\ \text { Louis Auslander } & \text { San Francisco } & \text { January } 1968 \\ \text { Rimhak Ree } & \text { Los Angeles } & \text { March } 1968 \\ \text { Irving Reiner } & \text { Chicago } & \text { April 1968 } \\ \text { Stephen Smale } & \text { Chicago } & \text { April 1968 } \\ \text { René Thom } & \text { Chicago } & \text { April } 1968 \\ \text { Hans F. Weinberger } & \text { Chicago } & \text { April 1968 } \\ \text { William Bade } & \text { Portland, Ore. } & \text { June 1968 } \\ \text { W. A. J. Luxemburg } & \text { Portland, Ore. } & \text { June 1968 }\end{array}$

An invitation to present Colloquium Lectures in 1968 was accepted by D. C. Spencer. The invitation marks the return to a former Society policy of presenting two sets of Colloquium Lectures at a Summer Meeting. John Milnor had previously accepted an invitation to present Colloquium Lectures in the Summer of 1968.

The Council approved the report of the Nominating Committee, resulting in the following slate for the October 1967 election:

President Elect: O. Zariski.

Vice Presidents: (two to be elected) David Blackwell, K. O. Friedrichs, Leo Zippin, D. C. Spencer.

Associate Secretaries: P. T. Bateman, R. S. Pierce.

Bulletin Editorial Committee: Gian-Carlo Rota.

Proceedings Editorial Committee: W. H. J. Fuchs, E. Michael, P. E. Thomas.

Colloquium Editorial Committee: N. E. Steenrod.

Mathematical Reviews Editorial Committee: R. S. Pierce.

Mathematical Surveys Editorial Committee: E. H. Brown, Jr.

Transactions and Memoirs Editorial Committee: J. J. Kohn, Dana Scott.

American Journal of Mathematics: S. Smale. 
Committee on Printing and Publishing: L. Auslander.

Council Members-at-large: (five to be elected) A. Borel, R. Bott, C. Davis, A. Douglis, I. Herstein, P. Hilton, S. Lang, A. Nijenhuis, R. Phillips, J. Schwartz.

Trustee: Irving Kaplansky.

Upon recommendation of the Nominating Committee, the Council elected Reese T. Prosser and F. Spitzer to represent the Society on the Editorial Board of the Duke Journal for the period 1968 through 1970.

Following a recommendation of the Committee to Monitor Problems in Communication, the Council approved the establishment of a service for the selective distribution of offprints of journal articles. In announcing this action to the membership, the Secretary wishes to emphasize that this is a distribution service and not a publication service. It does not replace journals but does enable a subscriber to the service quickly to obtain offprints of papers in areas of his previously expressed interest.

Editorial positions for several prospective publications were filled as follows:

Richard Bellman and Garrett Birkhoff as editors of the volume on Transport Theory in the series Proceedings of Symposia in Applied Mathematics (PSAM). The Symposium took place in April 1967.

G. B. Dantzig and Arthur F. Veinott, Jr. as editors of the volume on Mathematics in the Decision Sciences in PSAM. The Seminar took place in the Summer of 1967.

Alex Heller as editor of the volume on Applications of Categorical Algebra. The Symposium has been established for April 1968.

Garrett Birkhoff as editor of the volume on Numerical Solutions of Field Problems in Continuum Physics in PSAM. The Symposium has been established for April 1968 in connection with the Army Research Office (Durham) twentieth anniversary.

The Committee on Mathematics in the Life Sciences has, by mutual agreement, been made a joint committee of the Society with the Society for Industrial and Applied Mathematics.

The Council passed the following resolution about the documentation of work performed on federal government grants and contracts:

The Council of the American Mathematical Society urges responsible university officers to take immediate action to have Time and Effort Reports and similar documents pertaining to faculty members' time eliminated, because it considers that such documents are incompatible with academic life and work. The Council reiterates the traditional view that teaching and 
research are inseparable, and that accounting procedures in universities must take account of their unitary character.

The Council instructed the Secretary to distribute this resolution widely. The Council also instructed the President to appoint a committee to work toward mutually acceptable modifications with appropriate government administrators.

Professor W. J. LeVeque, as a member of the Society and representing a group of members, presented a proposal concerning a mathematical research and conference center. The feasibility and desirability of a conference center were discussed. The following resolution was adopted:

A mathematical research and conference center, equally available to all mathematical disciplines, would be of considerable value to the mathematical community on this continent.

The Council adjourned at 11:51 P.M.

There was a Business Meeting in Convocation Hall at 10:15 A.M. on Thursday, August 31.

The Secretary reported various actions by the Council. He noted that the new building for Society headquarters is to be occupied in October and to be dedicated at the same time, December 5-7, 1967, as the Conference on Communication Problems in the Mathematical Sciences. He noted that the Council has endorsed the 1940 Statement of Principles by the American Association of University Professors on Academic Freedom and Tenure.

The following amendment to the By-laws, already approved by the Council at its meeting in April of 1967 and announced in the call for the business meeting, was presented to the membership for action:

Alter the second sentence in Section 1, Article VII by the addition of the italicized words: "The term of office in the case of members of Publication Committees shall be four years for the Proceedings and Transactions (except for the years 1967 through 1970) and three years for the remaining committees, except that when the size of a publications committee is changed, the Council may authorize the election of a member for a shorter term."

Add a section $1 a$ as follows:

Section 1a. For the years 1967 through 1970, the terms of members of the Editorial Committee of the Transactions and Proceedings shall be as follows:

Transactions:
Term Expires December 31

1969

1969

1970

1970 
Proceedings:

Term Expires December 31

$$
\begin{aligned}
& \text { R. C. Buck } \\
& \text { A. P. Mattuck } \\
& \text { Irving Reiner } \\
& \text { W. R. Wasow }
\end{aligned}
$$

1967

1969

1969

1970

On January 1,1971, this section, as well as the parenthetical phrase (except for the years 1967 through 1970) of section 1 will be repealed.

The amendment was approved.

The business meeting adjourned at 10:40 A.M.

Everett Pitcher

Secretary

HERBERT FEDERER

Associate Secretary

Bethlehem, Pennsylvania

Providence, Rhode Island 